\title{
Left Out in the Cold
}

\author{
Justin Berk, MD, MPH, MBA*, Andrew PJ Olson, MD², Rabih M Geha, MD,4, Anand Patel, MD ${ }^{5}$, Reza Manesh, MD ${ }^{6}$
}

This icon represents the patient's case. Each paragraph that follows represents the discussants' thoughts.

${ }^{1}$ Departments of Medicine and Pediatrics, Warren Alpert School of Medicine at Brown University, Providence, Rhode Island; ${ }^{2}$ Departments of Medicine and Pediatrics, University of Minnesota Medical School, Minneapolis, Minnesota; ${ }^{3}$ Department of Medicine, University of California San Francisco, San Francisco, California; ${ }^{4}$ Medical Service, San Francisco VA Medical Center, San Francisco, California; ${ }^{5}$ Section of Hematology-Oncology, Department of Medicine, University of Chicago, Chicago, Illinois; ${ }^{\complement}$ Department of Internal Medicine, Johns Hopkins Hospital, Baltimore, Maryland.

A previously healthy 4-year-old boy presented to his pediatrician for nasal congestion, left ear pain, and intermittent fevers, which he'd been experiencing for 2 days. His exam was consistent with acute otitis media. Cefdinir was prescribed given a rash allergy to amoxicillin. His fever, congestion, and otalgia improved the next day.

Three days later he developed abdominal pain, fever, and labored breathing; his mother brought him to the emergency department (ED). His temperature was $38.0{ }^{\circ} \mathrm{C}$, heart rate 141 beats per minute, blood pressure $117 / 71 \mathrm{~mm} \mathrm{Hg}$, respiratory rate 22 breaths per minute; he had oxygen saturation of $96 \%$ on ambient air. Despite mild accessory muscle use, he appeared comfortable and interactive. His left tympanic membrane was bulging without erythema. His neck was supple and mucous membranes moist. He had neither cervical lymphadenopathy nor conjunctival pallor. The cardiopulmonary exam was normal except for tachycardia. His abdomen was soft and not distended without organomegaly or tenderness.

Upper respiratory tract symptoms are commonly encountered in pediatrics and most often result from self-limited viral processes. Evaluation of a child with upper respiratory tract symptoms aims to identify serious causes like meningitis, as well as assessing the need for antimicrobial therapy. Supportive management is often appropriate in otitis media. His new, more concerning symptoms portend either a progression of the original process causing his upper respiratory tract symptoms or a separate etiology. It is key to determine which signs and symptoms are associated with the primary process and which are compensatory or secondary. If he were to be more ill appearing, for example, it is possible that his respiratory distress may be related to an underlying systemic illness rather than a primary lung process. Respiratory distress, abdominal pain, and fever could be a result of sepsis from an intrabdominal process such as ruptured appendicitis, intussusception, or mal-

*Corresponding Author: Justin Berk, MD, MPH, MBA; Email: justin_berk@ brown.edu; Telephone: 401-444-6118; Twitter: @justinberk.

Published online first August 19, 2020.

Received: January 24, 2020; Revised: April 18, 2020; Accepted: April 28, 2020

๑ 2020 Society of Hospital Medicine DOI 10.12788/jhm.3453 rotation with volvulus. Other causes of sepsis, such as meningitis or severe mastoiditis, both rare complications of otitis media, should be considered, although he does not appear severely ill. Acute myelogenous leukemia or other malignancies and illnesses associated with immunodeficiency can present with sepsis and chloromas in the middle ear that can be misconstrued as otitis media.

A chest radiograph demonstrated left lower lobe patchy opacities concerning for pneumonia. Rapid respiratory syncytial virus and influenza antigen test results were negative. Laboratory testing for general bloodwork was not obtained. He was administered a single dose of intramuscular ceftriaxone, prescribed a 5-day course of azithromycin, and discharged home. The child's breathing gradually improved, but he continued to have subjective fevers. Two days later, he developed dark red urine. His mother brought him back to the outpatient clinic.

At the time of the ED visit, a diagnosis of community-acquired pneumonia was plausible given fever, mildly increased work of breathing, and an opacification on chest radiography. Most community-acquired pneumonia is caused by viruses; common bacterial causes for his age include Streptococcus pneumoniae and Moraxella catarrhalis. The first-line treatment for uncomplicated community-acquired pneumonia in children is amoxicillin, but this was appropriately avoided given his allergy.

The persistent fevers are surprising. The improvement in breathing corresponds to the treatment (and resolution) of community-acquired pneumonia. However, the development of dark urine does not. Red urine-in the absence of ingested pigments (such as those found in beets)—usually results from hematuria, hemoglobinuria, or myoglobinuria. Gross hematuria can originate from the kidneys to the urethral meatus. Abdominal masses, kidney trauma, or underlying kidney disease may all present with gross hematuria (or microscopic hematuria, seen only on urinalysis). The urine should be examined for the presence of heme, protein, and for evidence of infection; microscopy should be performed to examine for cellular casts and dysmorphic red cells. Tests of renal function, a comprehensive metabolic panel, evaluation of hematologic indexes, and assessments of inflammatory markers should be performed. 
The child lived with his parents and had no siblings. He experienced no physical trauma, and there was no family history of kidney disease or hematuria. His father had a persistent cough and fever for 1 month, but recovered around the time the patient began to experience his initial symptoms. This was the patient's third diagnosis of pneumonia. $\mathrm{He}$ had not traveled and was up to date with immunizations. He attended day care.

The fact that this is not the first episode of "pneumonia" raises important possibilities. The most likely one is that the child has had multiple viral infections; however, he could have an underlying primary immunodeficiency (PI) that predisposes him to recurrent infections. More severe Pls often present with recurrent sepsis, bacteremia, and failure to thrive, none of which were present in this case. Less severe Pls (such as selective IgA deficiency) could be possible. Another possibility is that these recurrent episodes of pneumonia are a relapsing and remitting noninfectious process, such as an antineutrophil cytoplasmic antibodies-associated vasculitis or anti-glomerular basement membrane disease. The patient's father's recent prolonged respiratory symptoms may be suggestive of pertussis or a "walking pneumonia" potentially caused by Mycoplasma or another atypical bacterium.

His temperature was $36.9^{\circ} \mathrm{C}$, heart rate 107 beats per minute, blood pressure was $106 / 67 \mathrm{~mm} \mathrm{Hg}$, and respiratory rate was 24 breaths per minute with oxygen saturation of $100 \%$ on ambient air. He was well appearing. His mucous membranes were moist, and oropharynx was clear. He had scleral icterus. The cardiopulmonary exam was normal. $\mathrm{He}$ had no significant lymphadenopathy, hepatosplenomegaly, or rashes.

The finding of jaundice is an important diagnostic pivot point, especially when combined with hematuria. The next step is determining if the jaundice is resulting from unconjugated or conjugated hyperbilirubinemia; the former most often stems from hemolysis or impairment in conjugation, while the latter results from intrahepatic or extrahepatic biliary defects. Tests for hepatobiliary injury including evaluations of alanine and aspartate aminotransferases and alkaline phosphatase, as well as for hepatic function such as tests of coagulation, should be performed.

The patient was referred to the ED and admitted for further evaluation. A complete blood count revealed a white blood cell (WBC) count of 10,700/ $\mu \mathrm{L}(61 \%$ polymorphonuclear neutrophils, 30\% lymphocytes, 5\% monocytes, $3 \%$ eosinophils, $1 \%$ basophils), hemoglobin count was $10.3 \mathrm{~g} / \mathrm{dL}$ (reticulocyte $2 \%$ with absolute reticulocyte count $58,400 / \mu \mathrm{L})$, and platelet count was $265,000 / \mu \mathrm{L}$. Components of the basic metabolic panel were within reference ranges except for a mildly elevated blood urea nitrogen level of $14 \mathrm{mg} / \mathrm{dL}$ with normal creatinine level of $0.3 \mathrm{mg} / \mathrm{dL}$. Total protein was $6.7 \mathrm{~g} / \mathrm{dL}$ (reference range, 6.4-8.3) and albumin
$3.9 \mathrm{~g} / \mathrm{dL}$ (reference range, 3.4-4.8). Alkaline phosphatase level was $188 \mathrm{U} / \mathrm{L}$ (reference range, 44-147), aspartate aminotransferase level $76 \mathrm{U} / \mathrm{L}$ (reference range, 0-40), and alanine aminotransferase level $12 \mathrm{U} / \mathrm{L}$ (reference range, 7-40). Total bilirubin level was $2.4 \mathrm{mg} / \mathrm{dL}$ (reference range, less than 1.5) with direct bilirubin level of $0.4 \mathrm{mg} / \mathrm{dL}$. His C-reactive protein level was $1.5 \mathrm{mg} / \mathrm{mL}$ (reference range, 0-0.75). Creatinine kinase (CK) level was 2,550 U/L (reference range, 2-198). International Normalized Ratio (INR) was 1.0. Urinalysis was notable for 2+ proteinuria, large hemoglobin pigment, and 6 red blood cells per high power field (reference range, $0-4$ ).

His blood urea nitrogen is elevated, reflecting either prerenal azotemia or increased absorption of nitrogenous products. Unconjugated hyperbilirubinemia may result from impaired hepatic bilirubin uptake (such as in heart failure or portosystemic shunts), impaired bilirubin conjugation (resulting from genetic conditions or drugs), or excess bilirubin production (such as in hemolysis); his anemia and lack of other evidence of hepatic dysfunction point to hemolysis as the etiology. The reticulocyte production index is approximately $1 \%$, which suggests that an increase in erythrocyte generation is present but inadequate. This, however, does not mean that an erythrocyte production abnormality is present since reticulocytosis can be delayed in many cases of acute hemolytic anemia. It is also possible that the same hemolytic process is affecting mature and immature erythrocytes. A peripheral blood smear should be reviewed for evidence of intravascular hemolysis and testing for autoimmune hemolysis should be performed. Notably, his white blood cell and platelet counts are preserved, which makes a bone marrow-involved malignancy or infiltrative process less likely. The alkaline phosphatase elevation may result from either intrahepatic or extrahepatic biliopathy; bone damage is also possible. The elevation of aspartate aminotransferase, CK, and potassium, along with marked urinary heme pigment, may indicate muscle damage; the most common myositis in children is benign acute childhood myositis resulting from viral infection. However, the moderate level of CK elevation seen in this case is nonspecific and can result from many different etiologies. A metabolic myopathy, such as carnitine palmitoyltransferase II deficiency, can be made worse by metabolic stress and result in rhabdomyolysis; the presentations of inborn errors of metabolism are varied and a planned-out, stepwise approach in evaluation is fundamental.

Lactic acid dehydrogenase (LDH) level was 1,457 U/L (reference range, 140-280), and haptoglobin level was less than $6 \mathrm{mg} / \mathrm{dL}$ (reference range, 30-200). Peripheral blood smear demonstrated occasional atypical, reactive-appearing lymphocytes with red cell clumping and agglutination, as well as rare target, burr, and fragmented red cells. Test results for urine myoglobin were negative. Results of urine culture were negative. No blood culture was collected.

The elevated LDH, decreased haptoglobin, and findings on the peripheral blood smear confirm hemolysis. The clumping 
of erythrocytes can be artifactual in the preparation of peripheral smears, but when considered in the context of hemolysis, may be clinically important. Clumping of erythrocytes on the peripheral smear indicates the binding of a protein to antigens on the erythrocyte membrane; when this occurs below body temperature, this is consistent with the presence of a "cold agglutinin," usually an lgM antibody directed at erythrocyte surface antigens that causes agglutination and destruction, especially in cooler areas of the body. This is a well-known complication of Mycoplasma pneumoniae infections as well as Epstein-Barr virus (EBV) infections; it may also occur with lymphoid malignancies or autoimmune disease.

Direct Coombs lgG test findings were negative, direct Coombs C3 test was positive, and direct Coombs polyspecific test was positive. M pneumoniae lgG antibody level was $1.4 \mathrm{mg} / \mathrm{dL}$ (reference ranges: <0.9, negative; 0.91-1.09, equivocal; >1.1, positive); $M$ pneumoniae lgM level was $529 \mathrm{U} / \mathrm{mL}$ (reference range: $<770$, negative). EBV capsid IgM and IgG levels were undetectable. EBV nuclear antigen lgG level was also undetectable. EBV viral load was fewer than 10 copies/mL. Antinuclear antibodies (ANA) level was negative. General lgE and IgM levels were normal, at 11 and $81 \mathrm{mg} / \mathrm{dL}$, respectively. Repeat complete blood count showed WBC of $7,800 / \mu \mathrm{L}$, hemoglobin of $8.7 \mathrm{~g} / \mathrm{dL}$, and platelet count of $341,000 / \mu \mathrm{L}$. The patient's hemoglobin remained stable during hospitalization.

This directed testing is helpful in further classifying the patient's hemolytic anemia. Autoimmune hemolytic anemias are classified into warm antibody-mediated, cold antibody-mediated, and mixed-type forms; drug-induced and alloimmune hemolytic anemias also occur. In addition, both systemic lupus erythematosus and antiphospholipid antibody syndrome can have hemolytic anemia with variable Coombs testing results; neither fit well in this case. The absence of red blood cell-directed IgG antibodies substantially decreases the likelihood of warm antibody-mediated hemolytic anemia. In cold antibodymediated hemolytic anemia, antibodies bind to the erythrocyte membrane and then adhere to complement C3, which leads to both intravascular and extravascular hemolysis. Important types of cold antibody-mediated hemolytic anemia in children are primary and secondary cold agglutinin disease, along with paroxysmal cold hemoglobinuria. The Donath-Landsteiner test can be helpful in differentiating these conditions. Antibodies to Mycoplasma may be delayed in response to acute infection, and a child who is reinfected may only produce lgG antibodies. Given the patient's clinical stability and previous health, the most likely diagnosis is Mycoplasma-induced cold antibody-mediated hemolytic anemia. It may be helpful to check convalescent titers to Mycoplasma in 2 to 4 weeks.

Donath-Landsteiner (D-L) antibody test results were positive. Medication-derived hemolytic anemia testing was conducted, but the presence of positive D-L antibody makes the test results inconclusive. This ultimately led to a diagnosis of paroxysmal cold hemoglobinuria $(\mathrm{PCH})$, presumably triggered by a viral syndrome. Convalescent titers to Mycoplasma were not checked given clinical improvement. Because the patient's hemoglobin was stable during hospitalization, he was not treated with steroids. His parents were counseled on avoiding cold temperatures for several days. Within 1 month, his hemoglobin had recovered without further evidence of hemolysis.

\section{DISCUSSION}

Hemolytic anemia refers to the accelerated destruction of red blood cells and can be further classified as acquired or hereditary. ${ }^{1}$ Hereditary conditions causing hemolytic anemia include enzymopathies (eg, glucose-6-phosphate dehydrogenase deficiency), hemoglobinopathies (eg, sickle cell disease), and membrane abnormalities (eg, hereditary spherocytosis). Acquired pathologies include microangiopathic hemolytic anemia (MAHA), anemias directly caused by certain infections such as malaria, and immune-mediated (Coombs-positive) hemolytic anemias.

MAHA can sometimes be life-threatening and is therefore important to identify quickly. In the right clinical context, such processes may be rapidly recognized by the presence of schistocytes on blood smear in addition to an elevated serum LDH level. Schistocytes suggest mechanical destruction of erythrocytes in the vasculature, the hallmark of MAHA. Important MAHAs include thrombocytopenic purpura, hemolytic-uremic syndrome, and disseminated intravascular coagulation. Though this patient did have a mildly elevated LDH, MAHA was less likely because there were no schistocytes on the blood smear.

Autoimmune hemolytic anemias (AlHAs) are another important subset of acquired hemolytic anemias. AlHAs occur when there is antibody-mediated destruction of erythrocytes. The direct Coombs test evaluates for antibody- or complementcoated erythrocytes. After administration of anti-lgG and antiC3 serum, the test evaluates for agglutination of the red cells caused by attached antibodies or complement. Coombspositive AlHA can also be categorized by the temperature of agglutination. "Warm" hemolysis often involves IgG autoantibodies (ie, warm agglutinins), while "cold" antibodies, usually IgM autoantibodies, bind at colder temperatures $\left(0-4{ }^{\circ} \mathrm{C}\right)$ and activate complements, including $\mathrm{C} 3$. In this patient, the Coombs C3 was positive while the Coombs IgG was negative, which is more suggestive of a cold complement-mediated pathway.

Cold AlHA can be further categorized into primary cold agglutinin disease, secondary cold agglutinin disease, and $\mathrm{PCH}$. Primary cold agglutinin disease is an autoimmune disorder that mostly occurs in adults. Secondary cold AlHA can often be triggered by bacterial infection (commonly $M$ pneumoniae) or viruses including EBV, measles, and mumps. ${ }^{2}$ Medications, including penicillin and cephalosporins, can also be implicated. Secondary cold AlHA is also linked with autoimmune diseases, such as systemic lupus erythematosus and lymphoproliferative disorders. PCH can be identified with the unique presence of a specific autoantibody (ie, D-L autoantibody) that agglutinates at cold temperatures but dissociates on subsequent rewarm- 
ing. ${ }^{3}$ Complement remains affixed and activates hemolysis.

The D-L antibody responsible for $\mathrm{PCH}$ is an $\lg \mathrm{G}$ antibody to the P-antigen present on the erythrocyte surface. Since the Coombs test is conducted at normal temperature, it will be positive for the affixed complement but not for lgG. The underlying mechanism for $\mathrm{PCH}$ was proposed by Julius Donath, MD, and Karl Landsteiner, MD, in 1904 and is considered to be the first description of autoimmune disease being precipitated by antibodies. ${ }^{4}$ The D-L antibody test itself is uncommonly performed and somewhat difficult to interpret, particularly in adults, and may lead to false-negative results. ${ }^{5}$

$\mathrm{PCH}$ is an acquired, cold AlHA more common to children ${ }^{6,7}$ and may account for up to $33 \%$ of pediatric AlHA cases. ${ }^{8}$ Typical presentation is after an upper respiratory tract illness; however, the trigger is often not identified. Implicated triggers include a number of viruses. ${ }^{9}$ Clinical presentation includes findings of intravascular hemolysis similar to those in our patient. The pathogenic IgG autoantibody is polyclonal and is likely formed because of immune stimulation, which is consistent with the predominance of nonmalignant triggers of this disease process. ${ }^{10}$ Hemolysis and associated symptoms are often exacerbated with cold exposure; both typically resolve within 2 weeks. In recurrent cases, which are a minority, immunosuppression may be considered. ${ }^{10}$

$\mathrm{PCH}$ remains an often-understated cause of hemolytic anemia particularly in children. Lacking obvious pathognomonic clinical symptoms, it may be overlooked for other forms of AlHA or MAHA. However, with a structured approach to evaluation, as with this patient who had hematuria and jaundice, early diagnosis can prevent an unnecessarily extensive workup and can provide reassurance to patient and parents. By understanding the basic categories of hemolytic anemia, the relevant blood testing available, and interpretation of Coombs test results, clinicians can ensure that $\mathrm{PCH}$ is a diagnosis that is not left out in the cold.

\section{KEY TEACHING POINTS}

- Examination for schistocytes on a blood smear can help identify life-threatening causes of hemolytic anemia.

- Characterization of cold AIHA includes defining the underlying etiology as primary cold agglutinin disease, secondary cold agglutinin disease, or $\mathrm{PCH}$.

- $\mathrm{PCH}$ is a cold AlHA that is an underrecognized cause of hemolytic anemia in children. The diagnosis of $\mathrm{PCH}$ is made by testing for the presence of the D-L antibody.

Disclosures: Dr Patel reported receiving an honorarium from the Human Diagnosis Project. The other authors reported having nothing to disclose.

\section{References}

1. Dhaliwal G, Cornett PA, Tierney LM Jr. Hemolytic anemia. Am Fam Physician. 2004;69(11):2599-2606

2. Djaldetti M. Paroxysmal cold hemoglobinuria. CRC Crit Rev Clin Lab Sci. 1978;9(1):49-83. https://doi.org/10.3109/10408367809150915

3. Levine P, Celano MJ, Falkowski F. The specificity of the antibody in paroxysmal cold hemoglobinuria (P.C.H.). Transfusion. 1963;3(4):278-280. https://doi. org/10.1111/j.1537-2995.1963.tb04643.x

4. Donath J, Landsteiner K. Uber Paroxysmale Hamoglobinurie. Munch Med Wochenschr. 1904;51:1590-1593

5. Zeller MP, Arnold DM, Al Habsi K, et al. Paroxysmal cold hemoglobinuria: a difficult diagnosis in adult patients. Transfusion. 2017;57(1):137-143. https:// doi.org/10.1111/trf.13888

6. Göttsche B, Salama A, Mueller-Eckhardt C. Donath-Landsteiner autoimmune hemolytic anemia in children. a study of 22 cases. Vox Sang. 1990;58(4):281286. https://doi.org/10.1111/j.1423-0410.1990.tb05000.x

7. Sokol RJ, Booker DJ, Stamps R. Erythropoiesis: paroxysmal cold haemoglobinuria: a clinico-pathological study of patients with a positive DonathLandsteiner test. Hematology. 1999;4(2):137-164. https://doi.org/10.1080/10 245332.1999.11746439

8. Petz LD. Cold antibody autoimmune hemolytic anemias. Blood Rev. 2008;22(1):1-15. https://doi.org/10.1016/j.blre.2007.08.002

9. Leibrandt R, Angelino K, Vizel-Schwartz M, Shapira I. Paroxysmal cold hemoglobinuria in an adult with respiratory syncytial virus. Case Rep Hematol. 2018;2018:1-3. https://doi.org/10.1155/2018/7586719

10. Gertz MA. Management of cold haemolytic syndrome. Br J Haematol. 2007;138(4):422-429. https://doi.org/10.1111/j.1365-2141.2007.06664.x 